Article

\title{
Applications of Canonical Quantum Gravity to Cosmology
}

\author{
Claus Gerhardt (D) \\ Ruprecht-Karls-Universität, Institut für Angewandte Mathematik, Im Neuenheimer Feld 205, \\ 69120 Heidelberg, Germany; gerhardt@math.uni-heidelberg.de
}

Received: 30 June 2019; Accepted: 2 August 2019; Published: 3 August 2019

check for updates

\begin{abstract}
We applied quantum gravitational results to spatially unbounded Friedmann universes and tried to answer some questions related to dark energy, dark matter, inflation, and the missing antimatter.
\end{abstract}

Keywords: quantization of gravity; quantum gravity; Friedmann universe; dark-energy density; dark matter; inflation; missing antimatter; cosmology; negative cosmological constant.

\section{Introduction}

The quantization of gravity is one of the most challenging open problems in physics. The Einstein equations are the Euler-Lagrange equations of the Einstein-Hilbert functional, and quantization of a Lagrangian theory requires to switch from a Lagrangian to a Hamiltonian view. In a groundbreaking paper, Arnowitt, Deser, and Misner [1] expressed the Einstein-Hilbert Lagrangian in a form that allowed to derive a corresponding Hamilton function by applying Legendre transformation. However, since the Einstein-Hilbert Lagrangian is singular, the Hamiltonian description of gravity is only correct if two additional constraints are satisfied, namely, the Hamilton constraint and the diffeomorphism constraint. Dirac [2] proved how to quantize a constrained Hamiltonian system, at least in principle, and his method has been applied to the Hamiltonian setting of gravity, cf. the paper by DeWitt [3] and the monographs by Kiefer [4] and Thiemann [5]. In the general case, when arbitrary globally hyperbolic spacetime metrics are allowed, the problem turned out to be extremely difficult, and solutions could only be found by assuming a high degree of symmetry, cf., e.g., [6].

However, in the papers $[7,8]$ we developed a model for the quantization of gravity for general hyperbolic spacetimes. In these papers, we eliminated the diffeomorphism constraint by reducing the number of variables and proving that the Euler-Lagrange equations for this special class of metrics were still the full Einstein equations. The Hamiltonian description of the Einstein-Hilbert functional then allowed canonical quantization.

We quantized the action by looking at the Wheeler-DeWitt equation in fiber bundle $E$, where the base space is a Cauchy hypersurface of the spacetime that was quantized and the elements of the fibers are Riemannian metrics. The fibers of $E$ are equipped with a Lorentzian metric, such that they are globally hyperbolic, and the transformed Hamiltonian, which is now a hyperbolic operator $\hat{H}$, is a normally hyperbolic operator only acting in the fibers. The Wheeler-DeWitt equation has the form $\hat{H} u=0$ with $u \in C^{\infty}(E, \mathbb{C})$, and we defined a symplectic vector space and a corresponding Weyl system with the help of Green's operator.

The Wheeler-DeWitt equation seems to be the obvious quantization of the Hamilton condition. However, $\hat{H}$ acts only in the fibers and not in the base space, which is due to the fact that the derivatives are only ordinary covariant derivatives and not functional derivatives. Though they are supposed to be functional derivatives, this property is not really invoked when a functional derivative is applied to $u$, since the result is the same as applying a partial derivative. 
Therefore, we discarded the Wheeler-DeWitt equation in the paper [9] and also in the monograph [10], and expressed the Hamilton condition differently by looking at the evolution equation of the mean curvature of the foliation hypersurfaces $M(t)$ and implementing the Hamilton condition on the right-hand side of this evolution equation. We replaced the left-hand side, a time derivative, by the corresponding Poisson brackets. After canonical quantization, the modified evolution equation was transformed to an equation satisfied by operators that acted on functions $u \in C^{\infty}(E, \mathbb{C})$.

Since the Poisson brackets became a commutator, we could employ the fact that derivatives are functional derivatives since we had to differentiate the scalar curvature of a metric when we applied the operator equation to a smooth function and tried to simplify the resulting equation. As a result of the simplification of the commutator action, we obtained an elliptic differential operator in the base space, the main part of which was the Laplacian with respect to a fiber element. Here, we considered functions $u$ depending on variables $\left(x, g_{i j}\right)$, where $x$ is a point in the base space $\mathcal{S}_{0}, x \in \mathcal{S}_{0}$, and $g_{i j}$ is an element of the fibers. The fiber metrics have the form

$$
g_{i j}=t^{\frac{4}{n}} \sigma_{i j}
$$

where $0<t<\infty$ is a timelike fiber variable, which is referred to as time, $n \geq 3$, is the dimension of $\mathcal{S}_{0}$, and $\sigma_{i j}$ is a Riemannian metric, depending only on $x$, subject to the requirement

$$
\operatorname{det} \sigma_{i j}=\operatorname{det} \chi_{i j}
$$

cf. ([10] equs. (1.4.103) \& (1.4.104), p. 29) and also ([10], Remark 1.6.8). The arbitrary but fixed metric $\chi_{i j}$ in $\mathcal{S}_{0}$ was introduced to transform densities $\operatorname{det} g_{i j}$ to functions.

On the right-hand side of the evolution equation, the interesting term was $H^{2}$, the square of the mean curvature. It transformed to a second time derivative, the only remaining derivative with respect to a fiber variable, since differentiations with respect to the other variables canceled each other. The resulting quantized equation is then a wave equation in a globally hyperbolic spacetime

$$
Q=(0, \infty) \times \mathcal{S}_{0}
$$

of the form

$$
\frac{1}{32} \frac{n^{2}}{n-1} \ddot{u}-(n-1) t^{2-\frac{4}{n}} \Delta u-\frac{n}{2} t^{2-\frac{4}{n}} R u+n t^{2} \Lambda u=0,
$$

where $\mathcal{S}_{0}$ is a Cauchy hypersurface of the original spacetime and the Laplacian and scalar curvature $R$ are formed with respect to a metric $\sigma_{i j}$ satisfying (2), and $\Lambda$ is a cosmological constant. Function $u$ depends on $\left(x, t, \sigma_{i j}\right)$.

Since metric $\chi_{i j}$ is also a fiber metric, we may choose $\sigma_{i j}=\chi_{i j}$, and because it is also arbitrary, we may set $\chi_{i j}$ to be the original metric of Cauchy hypersurface $\mathcal{S}_{0}$, cf. ([10], Remark 1.6.8 on page 49). Function $u$ then only depends on $(t, x), u=u(t, x)$. For a detailed derivation of Equation (4), we refer to ([10], Chapter 1.6) or ([9], Section 6).

When $\mathcal{S}_{0}$ is a space of constant curvature then the wave equation, considered only for functions $u$ that do not depend on $x$, it is identical to the equation obtained by quantizing the Hamilton constraint in a Friedmann universe without matter, but including a cosmological constant, cf. ([10], Remark 1.6.11 on page 50) or ([9], Remark 6.11).

There exist temporal and spatial self-adjoint operators $H_{0}$ resp. $H_{1}$, such that the hyperbolic equation is equivalent to

$$
H_{0} u-H_{1} u=0,
$$

where $u=u(t, x)$. Operator $H_{0}$ is defined by

$$
H_{0} w=\varphi_{0}^{-1}\left\{-\frac{1}{32} \frac{n^{2}}{n-1} \ddot{w}-n t^{2} \Lambda w\right\}
$$


where $w=w(t), w \in C_{c}^{\infty}\left(\mathbb{R}_{+}, \mathbb{C}\right)$, and $\varphi_{0}=t^{2-\frac{4}{n}}$, while the definition of $H_{1}$ is given by

$$
H_{1} v=-(n-1) \Delta v-\frac{n}{2} R v
$$

where $v=v(x), v \in C_{c}^{\infty}\left(\mathcal{S}_{0}, \mathbb{C}\right)$. More precisely, operators $H_{i}, i=0,1$, are the corresponding unique self-adjoint extensions of the operators defined above in the appropriate function spaces.

Assuming $\Lambda<0$, we proved that $H_{0}$ has a pure point spectrum with positive eigenvalues $\lambda_{i}$, cf. ([10], Chapter 6.2), especially ([10], Theorem 6.2.5 on page 144), while, for $H_{1}$, it is possible to find corresponding eigendistributions for each of the eigenvalues $\lambda_{i}$, if $\mathcal{S}_{0}$ is asymptotically Euclidean or if the quantized spacetime is a black hole with a negative cosmological constant, cf. [11-13] or ([10], Chapters 3-5), and also if $\mathcal{S}_{0}$ is the hyperbolic space $\mathcal{S}_{0}=\mathbb{H}^{n}, n \geq 3$, cf. Section 6 on page 13.

Let $w_{i}, i \in \mathbb{N}$, be an orthonormal basis for the temporal eigenvalue problems

$$
H_{0} w_{i}=\lambda_{i} w_{i}
$$

and $v_{i}$ be corresponding smooth eigendistributions for spatial eigenvalue problems

$$
H_{1} v_{i}=\lambda_{i} v_{i}
$$

then

$$
u_{i}=w_{i} v_{i}
$$

are special solutions of the wave Equation (4).

Temporal eigenvalues $\lambda_{i}$ all have multiplicity 1, the spatial eigenvalues are the same eigenvalues, but they may have higher multiplicities. In the case of black holes, this is caused by very compelling intrinsic mathematical reasons, cf. ([10], Chapter 6.4), but unless there are either convincing intrinsic or extrinsic reasons, like data, we chose the spatial eigenspaces to be one-dimensional because spatial eigenvalues in general belong to the continuous spectrum of spatial Hamiltonian $H_{1}$. If $\mathcal{S}_{0}$ is the Cauchy hypersurface of a Friedmann universe, we only considered smooth spherically symmetric spatial eigenfunctions, which also leads to one-dimensional spatial eigenspaces, cf. ([10], Chapter 6.6) for the Euclidean case and Section 6 on page 13 for the hyperbolic case.

One can then define an abstract Hilbert space $\mathcal{H}$ spanned by the $u_{i}$ and a self-adjoint operator $H$, unitarily equivalent to $H_{0}$, such that

$$
H u_{i}=\lambda_{i} u_{i}
$$

$e^{-\beta H}$ is then of trace class in $\mathcal{H}$ for all $\beta>0$ and the canonical extension of $H$ to the corresponding symmetric Fock space $\mathcal{F}$, which is still called $H$, shares this property. Hence, we can define partition function $Z$,

$$
Z=\operatorname{tr} e^{-\beta H}
$$

the operator density

$$
\hat{\rho}=Z^{-1} e^{-\beta H}, \quad \forall \beta>0,
$$

the average energy and the von Neumann entropy in $\mathcal{F}$. Eigenvectors $u_{i}$ can also be viewed as elements of $\mathcal{F}$, and they are also then eigenvectors of $\hat{\rho}$.

In the present paper, we want to apply these quantum gravitational results to cosmology by looking at a Friedmann universe

$$
N=I \times \mathcal{S}_{0},
$$

where $\mathcal{S}_{0}$ is an $n$-dimensional simply connected space of constant curvature $\tilde{\kappa}$,

$$
\tilde{\kappa} \in\{0,-1\},
$$


i.e., $\mathcal{S}_{0}$ is either $\mathbb{R}^{n}$ or the hyperbolic space $\mathbb{H}^{n}, n \geq 3$. We tried to answer some questions related to dark energy, dark matter, inflation, and the missing antimatter. In doing so, we also show that assuming a negative cosmological constant is not a contradiction to the observational result of an expanding universe. Usually, a positive cosmological constant is supposed to be responsible for dark energy, and dark matter is sometimes explained by assuming so-called extended theories of gravity, confer, e.g., the papers $[14,15]$. In this paper, we rely on general relativity combined with some quantum gravitational ingredients.

Let us summarize the main result as a theorem, where $\rho_{\mathrm{dm}}$ resp. $\rho_{\mathrm{de}}$ refer to dark-matter resp. dark-energy densities, which we defined as eigenvalues of operator density $\hat{\rho}$ in $\mathcal{F}$, and $\rho_{3}$ is conventional density. $\mathrm{Z}$ is the partition function, $T>0$ absolute temperature, and $\lambda_{0}>0$ the smallest eigenvalue of the Hamiltonian $H$.

Theorem 1. Let cosmological constant $\Lambda$

$$
-1<\Lambda<0,
$$

be given, and consider the perfect fluid defined by density

$$
\rho=\rho_{\mathrm{dm}}+\rho_{\mathrm{de}}+\rho_{3}
$$

satisfying Assumptions (88), (89), (101) and (102). Moreover, we suppose that $\beta=T^{-1}$ and scale factor a are functions depending on $t$. The initial value problems

$$
\frac{\ddot{a}}{a}=-\frac{\kappa^{2}}{n(n-1)}\{(n-2) \rho+n p\}+\frac{2}{n(n-1)} \Lambda
$$

and

$$
\dot{\beta}=-n \frac{\rho_{\mathrm{dm}}}{\frac{\partial}{\partial \beta}\left(\rho_{\mathrm{dm}}+\rho_{\mathrm{de}}\right)} a^{-1} \dot{a} .
$$

with initial values $\left(\beta_{0}, a_{0}, \dot{a}_{0}\right)$ are then solvable in $I=\left[t_{0}, \infty\right)$ provided $\beta_{0}>0$ is so large that (39) on page 5 as well as

$$
\frac{2 \kappa^{2}}{n(n-1)} Z^{-1}\left\{1-\frac{1}{2}(n-2) \alpha_{0} e^{-\beta \lambda_{0}}\right\}+\frac{2}{n(n-1)} \Lambda>0
$$

are valid at $\beta=\beta_{0}$ and $a_{0}>0$ has to be chosen such that after adding

$$
-\frac{\kappa^{2}}{n(n-1)}\left(n\left(1+\omega_{3}\right)-2\right) \gamma_{3} a_{0}^{-n\left(1+\omega_{3}\right)}
$$

to the left-hand side of Inequality (20) the inequality still remains valid at $\beta=\beta_{0}$. Initial value $\dot{a}_{0}$ is supposed to be positive. Solution $(\beta, a)$ then satisfies

$$
\begin{aligned}
& \dot{\beta}>0, \\
& \dot{a}>0, \\
& \ddot{a}>0
\end{aligned}
$$

and

$$
\frac{2}{n(n-1)} \kappa^{2} \rho+\frac{2}{n(n-1)} \Lambda-\tilde{\kappa} a^{-2}>0 .
$$

In order that $(\beta, a)$ also satisfies the first Friedmann equation, $\dot{a}_{0}$ has to be chosen appropriately, namely, such that the first Friedmann equation is valid for $t=t_{0}$, which is possible, in view of (25).

Remark 1. Let us also mention that we use (modified) Planck units in this paper, i.e.,

$$
c=\kappa^{2}=\hbar=K_{B}=1,
$$


where $\kappa^{2}$ is the coupling constant connecting the Einstein tensor with the stress-energy tensor

$$
G_{\alpha \beta}+\Lambda \bar{g}_{\alpha \beta}=\kappa^{2} T_{\alpha \beta}
$$

\section{Dark-Energy Density}

In [10], Remark 6.5.5, we proposed to use the eigenvalue of density operator $\hat{\rho}$ with respect to vacuum vector $\eta$, which is $Z^{-1}$,

$$
\hat{\rho} \eta=Z^{-1} \eta,
$$

as the source of dark-energy density, and though this eigenvalue is the vacuum, or zero-point, energy, and many authors have proposed vacuum energy is responsible for dark energy, these proposals all assumed the cosmological constant to be positive, while we assume $\Lambda<0$ because of the spectral resolution of the wave equation; otherwise, the temporal Hamiltonian does not have a pure point spectrum. However, if $\Lambda<0$, then we have to assure that $Z^{-1}$ dominates $\Lambda$, which is only the case if

$$
T<T_{0}=T_{0}(|\Lambda|) .
$$

Note that $Z$ depends on eigenvalues $\lambda_{i}$ and on

$$
\beta=T^{-1} \text {. }
$$

First, we emphasize that we treat

$$
\rho_{\mathrm{de}}=Z^{-1}
$$

as a constant, i.e., we define the perfect fluid stress-energy tensor by

$$
T_{\alpha \beta}=-\rho_{\mathrm{de}} \bar{g}_{\alpha \beta}
$$

Let $\lambda_{i}>0, i \in \mathbb{N}$, be the eigenvalues of temporal Hamiltonian $H_{0}$ for a given $\Lambda<0$, and let $\bar{\lambda}_{i}$ be the eigenvalues for

$$
\Lambda=-1,
$$

then

$$
\lambda_{i}=\bar{\lambda}_{i}|\Lambda|^{\frac{n-1}{n}},
$$

cf. ([10], Lemma 6.4.9, p. 172), and define parameter $\tau$ by

$$
\tau=|\Lambda|^{\frac{n-1}{n}},
$$

where we now assume

$$
|\Lambda|<1,
$$

throughout the rest of the paper. We proved in [10], Theorem 6.5.6, p. 180, that

$$
\lim _{\tau \rightarrow 0} Z=\infty,
$$

or equivalently, that

$$
\lim _{\tau \rightarrow 0} \rho_{\mathrm{de}}=0 .
$$

However, we now derive a more precise estimate of $\rho_{\mathrm{de}}=Z^{-1}$ involving $\beta$ and $\Lambda$.

Lemma 1. For any $\Lambda$ satisfying $-1<\Lambda<0$, there exists exactly one $T_{0}>0$, such that

$$
Z^{-1}(\beta)>|\Lambda| \quad \forall \beta>\beta_{0}=T_{0}^{-1},
$$


where we recall that

$$
\beta=T^{-1} \text {. }
$$

Proof. In view of Equation (34) we deduce that

$$
Z(\beta) \equiv Z\left(\beta, \lambda_{i}\right)=\bar{Z}\left(\gamma, \bar{\lambda}_{i}\right) \equiv \bar{Z}(\gamma),
$$

where

$$
\gamma=\beta|\Lambda|^{\frac{n-1}{n}}
$$

From relations

$$
0<E=-\frac{\partial \log Z}{\partial \beta}=\frac{\partial \log Z^{-1}}{\partial \beta},
$$

cf. ([10], Equations (6.5.30) and (6.5.32), p. 176),

$$
\lim _{\beta \rightarrow \infty} Z(\beta)=1 \text {, }
$$

and

$$
\lim _{\beta \rightarrow 0} Z(\beta)=\infty,
$$

cf. ([10], Theorem 6.5.8, p. 181), we then conclude that there exists exactly one $\gamma_{0}$, such that

$$
\bar{Z}^{-1}\left(\gamma_{0}\right)=|\Lambda|
$$

and, furthermore, that

$$
\bar{Z}^{-1}(\gamma)>\bar{Z}^{-1}\left(\gamma_{0}\right) \quad \forall \gamma>\gamma_{0},
$$

completing the proof of the lemma.

Thus, defining dark-energy density by Equations (31) and (32), we immediately deduce:

Theorem 2. Let $T_{0}$ be the temperature defined in Lemma 1, and assume that temperature $T$ satisfies $T<T_{0}$; then, dark-energy density guarantees that the Friedmann universe with negative cosmological constant $\Lambda$,

$$
-1<\Lambda<0,
$$

is expanding, such that

$$
\dot{a}>0
$$

as well as

$$
\ddot{a}>0 .
$$

Proof. The Friedmann equations for a perfect fluid with energy $\rho$ and pressure $p$ are

$$
\frac{\dot{a}^{2}}{a^{2}}=\frac{2}{n(n-1)} \kappa^{2} \rho+\frac{2}{n(n-1)} \Lambda-\tilde{\kappa} a^{-2}
$$

and

$$
\frac{\ddot{a}}{a}=-\frac{\kappa^{2}}{n(n-1)}\{(n-2) \rho+n p\}+\frac{2}{n(n-1)} \Lambda .
$$

Choosing $\rho=\rho_{\mathrm{de}}$, we also specified

$$
p=-\rho_{\mathrm{de}}
$$


yielding

$$
\frac{\ddot{a}}{a}=\frac{2 \kappa^{2}}{n(n-1)} \rho_{\mathrm{de}}+\frac{2}{n(n-1)} \Lambda .
$$

Moreover, in our units,

$$
\kappa^{2}=1
$$

and we also only considered space forms satisfying

$$
\tilde{\kappa} \leq 0,
$$

hence, the theorem is proved in view of Lemma 1.

\section{Inflationary Period}

Immediately after the Big Bang, the development of the universe had to have been governed by quantum gravitational forces, i.e., by the eigenfunctions resp. eigendistributions of the corresponding temporal and spatial Hamiltonians, which we combined to a single Hamiltonian $H$ acting in an abstract separable Hilbert space $\mathcal{H}$ spanned by eigenvectors $u_{i}$

$$
H u_{i}=\lambda_{i} u_{i}
$$

where the eigenvalues, which all have multiplicity 1 , are ordered

$$
0<\lambda_{0}<\lambda_{1}<\cdots
$$

and converge to infinity

$$
\lim _{i \rightarrow \infty} \lambda_{i}=\infty .
$$

The dominant energies near the Big Bang are therefore eigenvalues

$$
\lambda_{i}=\left\langle H u_{i}, u_{i}\right\rangle
$$

for large $i$, and we assume, when considering the development of a Friedmann universe, that this development is driven by a perfect fluid

$$
T_{\alpha \beta}=-\rho_{i} \bar{g}_{\alpha \beta},
$$

where

$$
\rho_{i}=\lambda_{i}
$$

Looking at Friedmann equations

$$
\frac{\dot{a}^{2}}{a^{2}}=\frac{2 \kappa^{2}}{n(n-1)} \rho_{i}+\frac{2}{n(n-1)} \Lambda-\tilde{\kappa} a^{-2}
$$

and

$$
\frac{\ddot{a}}{a}=\frac{2 \kappa^{2}}{n(n-1)} \rho_{i}+\frac{2}{n(n-1)} \Lambda
$$

we conclude that the universe is expanding rapidly, depending on eigenvalue $\rho_{i}=\lambda_{i}$. The corresponding eigenvector, or particle, $u_{i}$ decays after some time and produces lower-order eigenvectors, or maybe particles that can be looked at as matter or radiation satisfying corresponding equations of state.

After some time, the inflationary period ends, and only the stable ground state $u_{0}$,

$$
H u_{0}=\lambda_{0} u_{0},
$$


together with conventional matter and radiation are responsible for the further development of the Friedmann universe.

Eigenvalue $\lambda_{0}$ is of the order $|\Lambda|^{\frac{n-1}{n}}$ in view of (34) on page 5; hence, it dominates $\Lambda$ for small values of $|\Lambda|$.

\section{Dark Matter}

Let $\hat{\rho}$ be the density operator acting in Fock space $\mathcal{F}$,

$$
\hat{\rho}=Z^{-1} e^{-\beta H},
$$

where we use the same symbol $H$ to denote self-adjoint operator $H$ in separable Hilbert space $\mathcal{H}$, as well its canonical extension to corresponding symmetric Fock space $\mathcal{F}_{+}(\mathcal{H}) \equiv \mathcal{F}$. In Section 2, we defined dark-energy density $\rho_{\text {de }}$ by

$$
\rho_{\mathrm{de}}=\langle\hat{\rho} \eta, \eta\rangle=Z^{-1}
$$

and we propose to define dark-matter density by

$$
\rho_{\mathrm{dm}}=\alpha_{0}\left\langle\hat{\rho} u_{0}, u_{0}\right\rangle=\alpha_{0} e^{-\beta \lambda_{0}} \mathrm{Z}^{-1},
$$

where $u_{0}$ is a unit eigenvector of $H$ satisfying

$$
H u_{0}=\lambda_{0} u_{0}
$$

and

$$
\alpha_{0}>1
$$

an otherwise arbitrary constant. Its presence should guarantee that there exists $\beta_{0}>0$, such that

$$
\frac{\partial}{\partial \beta}\left(\rho_{\mathrm{dm}}+\rho_{\mathrm{de}}\right)<0 \quad \forall \beta \geq \beta_{0},
$$

as we now prove:

Lemma 2. Let $\alpha_{0}$ satisfy (70) and $\Lambda$

$$
-1<\Lambda \leq \Lambda_{0}<0,
$$

then, there exists $\beta_{0}=\beta_{0}\left(\alpha_{0},\left|\Lambda_{0}\right|\right)$, such that Inequality (71) is valid.

Proof. In view (43) on page 6, we have

$$
\frac{\partial}{\partial \beta}\left(\rho_{\mathrm{dm}}+\rho_{\mathrm{de}}\right)=-\alpha_{0} \lambda_{0} e^{-\beta \lambda_{0}} Z^{-1}+\alpha_{0} e^{-\beta \lambda_{0}} Z^{-1} E+Z^{-1} E,
$$

where

$$
E=\sum_{i=0}^{\infty} \frac{\lambda_{i}}{e^{\beta \lambda_{i}}-1}=\frac{\lambda_{0}}{e^{\beta \lambda_{0}}-1}+\sum_{i=1}^{\infty} \frac{\lambda_{i}}{e^{\beta \lambda_{i}}-1},
$$

cf. ([10], Equation (6.5.32), p. 176) or simply differentiate. Hence, we obtain

$$
\begin{aligned}
E e^{\beta \lambda_{0}} & =\frac{\lambda_{0} e^{\beta \lambda_{0}}}{e^{\beta \lambda_{0}}-1}+\sum_{i=1}^{\infty} \frac{\lambda_{i}}{e^{\beta\left(\lambda_{i}-\lambda_{0}\right)}-e^{-\beta \lambda_{0}}} \\
& \leq \frac{\lambda_{0} e^{\beta \lambda_{0}}}{e^{\beta \lambda_{0}}-1}+\sum_{i=1}^{\infty} \frac{\lambda_{i}}{e^{\beta\left(\lambda_{i}-\lambda_{0}\right)}-1}
\end{aligned}
$$


and we conclude

$$
\lim _{\beta \rightarrow \infty} E e^{\beta \lambda_{0}}=\lambda_{0}
$$

since

$$
\begin{aligned}
\sum_{i=1}^{\infty} \frac{\lambda_{i}}{e^{\beta\left(\lambda_{i}-\lambda_{0}\right)}-1} & =\sum_{i=1}^{\infty} \frac{\lambda_{i}-\lambda_{0}}{e^{\beta\left(\lambda_{i}-\lambda_{0}\right)}-1}+\sum_{i=1}^{\infty} \frac{\lambda_{0}}{e^{\beta\left(\lambda_{i}-\lambda_{0}\right)}-1} \\
& \leq \sum_{i=1}^{\infty} \frac{\mu_{i}}{e^{\beta \mu_{i}}-1}+\lambda_{0}\left(\lambda_{1}-\lambda_{0}\right)^{-1} \sum_{i=1}^{\infty} \frac{\mu_{i}}{e^{\beta \mu_{i}}-1}
\end{aligned}
$$

where $\mu_{i}$ is defined by

$$
\mu_{i}=\lambda_{i}-\lambda_{0} \geq \lambda_{1}-\lambda_{0}>0 \quad \forall i \geq 1 .
$$

Thus, the right-hand side of Equation (77) is estimated from above by

$$
\left(1+\lambda_{0}\left(\lambda_{1}-\lambda_{0}\right)^{-1}\right) E\left(\beta, \mu_{i}\right)
$$

and

$$
\lim _{\beta \rightarrow \infty} E\left(\beta, \mu_{i}\right)=0,
$$

cf. ([10], Equation (6.5.71), p. 181). Furthermore, we know

$$
\lambda_{0}=\bar{\lambda}_{0}|\Lambda|^{\frac{n-1}{n}},
$$

cf. (34). Combining these estimates, we conclude that there exists

$$
\beta_{0}=\beta_{0}\left(\alpha_{0},\left|\Lambda_{0}\right|\right)
$$

such that

$$
\frac{\partial}{\partial \beta}\left(\rho_{\mathrm{dm}}+\rho_{\mathrm{de}}\right) \leq-\frac{\alpha_{0}-1}{2} \lambda_{0} e^{-\beta \lambda_{0}} Z^{-1} \quad \forall \beta \geq \beta_{0} .
$$

The limits in Equations (76) and (80) are also uniform in $|\Lambda|$ because of (72).

Dark matter is supposed to be dust, i.e., its pressure vanishes, and hence, $\rho_{\mathrm{dm}}$ cannot be constant, which is tantamount to

$$
\beta \not \equiv \text { const, }
$$

since we assume that $\Lambda$ is constant. Thus, $\rho_{\text {de }}$ is also not constant, though we still assume that its stress-energy tensor is defined by

$$
T_{\alpha \beta}=-\rho_{\operatorname{de}} \bar{g}_{\alpha \beta} .
$$

Therefore, we can only establish the continuity equation for

$$
\rho_{\mathrm{dm}}+\rho_{\mathrm{de}}
$$

and not separately for each density. Let a dot or a prime indicate differentiation with respect to time $t$; then, the continuity equation has the form

$$
\left(\rho_{\mathrm{dm}}+\rho_{\mathrm{de}}\right)^{\prime}=-n \rho_{\mathrm{dm}} a^{-1} \dot{a},
$$

because

$$
p_{\mathrm{dm}}=0
$$

and

$$
p_{\mathrm{de}}=-\rho_{\mathrm{de}}
$$


The left-hand side of Equation (87) is equal to

$$
\frac{\partial}{\partial \beta}\left(\rho_{\mathrm{dm}}+\rho_{\mathrm{de}}\right) \dot{\beta}
$$

and we see that the continuity equation can only be satisfied if

$$
\dot{\beta}=-n \frac{\rho_{\mathrm{dm}}}{\frac{\partial}{\partial \beta}\left(\rho_{\mathrm{dm}}+\rho_{\mathrm{de}}\right)} a^{-1} \dot{a} .
$$

From Lemma 2, we immediately derive

Lemma 3. Let the assumptions of Lemma 2 be satisfied and suppose that $\dot{a}>0$; then, for any solution $\beta=\beta(t)$ of (91) in interval

$$
I=\left[t_{0}, b\right), \quad t_{0}<b \leq \infty,
$$

with initial value

$$
\beta\left(t_{0}\right) \geq \beta_{0}
$$

inequality

$$
\dot{\beta}>0
$$

is valid and hence

$$
\beta(t) \geq \beta_{0} \quad \forall t \in I .
$$

Furthermore, $\dot{\beta}$ can be expressed in the form

$$
\dot{\beta}=n \delta\left(\alpha_{0}-1\right)^{-1} \alpha_{0} a^{-1} \dot{a},
$$

where $\delta=\delta\left(t, \beta_{0}\right)$ satisfies

$$
1 \leq \delta \leq 2
$$

and

$$
\lim _{\beta_{0} \rightarrow \infty} \delta=1,
$$

i.e.,

$$
\beta(t)-\beta\left(t_{0}\right) \approx n \delta \alpha_{0}\left(\alpha_{0}-1\right)^{-1}\left(\log a(t)-\log a\left(t_{0}\right)\right) .
$$

\section{Proof.}

“(94)" Follows from (71) and (91).

"(96)" To prove the claim, we combine (73), (91) and (76).

"(97)" and "(98)" — same argument as before.

"(99)" Obvious in view of (96) and (98).

Now, we are prepared to solve Friedmann Equations (51) and (52) on page 6 for

$$
\rho=\rho_{\mathrm{dm}}+\rho_{\mathrm{de}}+\rho_{3},
$$

where $\rho_{3}$ is a conventional density satisfying equation of state

$$
p_{3}=\omega_{3} \rho_{3}
$$

assuming

$$
\omega_{3}>-1 \text {. }
$$


$\rho_{3}$ is only added for good measure, and we are allowed to assume

$$
\rho_{3}=0,
$$

since its presence is not essential.

We also emphasize that we have to solve a third equation, namely, Equation (91). We solve the Friedmann equations and Equation (91) in the interval

$$
I=\left[t_{0}, \infty\right), \quad t_{0}>0,
$$

for unknown functions $(a, \beta)$ with prescribed positive initial values $\left(a_{0}, \dot{a}_{0}, \beta_{0}\right) . \beta_{0}$ can be arbitrary but large enough, such that the assumptions in Lemma 2 and Lemma 3 are satisfied. If $\rho_{3}$ vanishes then $a_{0}>0$ can be arbitrary, otherwise it has to be large enough. The last initial value $\dot{a}_{0}>0$ cannot be arbitrary, instead it has to be chosen such that the first Friedmann equation is initially valid at $t=t_{0}$.

If these assumptions are satisfied, then we solve Equations (52) on page 6 and (91). The first Friedmann equation is then automatically valid. For simplicity, we only consider the case

$$
\rho_{3}>0
$$

to avoid case distinctions. Then, we deduce from the continuity equation,

$$
\rho_{3}=\gamma_{3} a^{-n\left(1+\omega_{3}\right)},
$$

where $\gamma_{3}>0$ is a given constant.

Let us now prove:

Theorem 3. Let cosmological constant $\Lambda$

$$
-1<\Lambda<0,
$$

be given, and consider the perfect fluid defined by density

$$
\rho=\rho_{\mathrm{dm}}+\rho_{\mathrm{de}}+\rho_{3}
$$

satisfying Assumptions (88), (89), (101), and (102). Moreover, we suppose that $\beta=T^{-1}$ and scale factor a are functions depending on $t$. The initial value problems

$$
\frac{\ddot{a}}{a}=-\frac{\kappa^{2}}{n(n-1)}\{(n-2) \rho+n p\}+\frac{2}{n(n-1)} \Lambda
$$

and

$$
\dot{\beta}=-n \frac{\rho_{\mathrm{dm}}}{\frac{\partial}{\partial \beta}\left(\rho_{\mathrm{dm}}+\rho_{\mathrm{de}}\right)} a^{-1} \dot{a} .
$$

with initial values $\left(\beta_{0}, a_{0}, \dot{a}_{0}\right)$ are then solvable in $I=\left[t_{0}, \infty\right)$ provided $\beta_{0}>0$ is so large that (39) on page 5 as well as

$$
\frac{2 \kappa^{2}}{n(n-1)} Z^{-1}\left\{1-\frac{1}{2}(n-2) \alpha_{0} e^{-\beta \lambda_{0}}\right\}+\frac{2}{n(n-1)} \Lambda>0
$$

are valid at $\beta=\beta_{0}$ and $a_{0}>0$ has to be chosen such that after adding

$$
-\frac{\kappa^{2}}{n(n-1)}\left(n\left(1+\omega_{3}\right)-2\right) \gamma_{3} a_{0}^{-n\left(1+\omega_{3}\right)}
$$


to the left-hand side of (111) the inequality still remains valid at $\beta=\beta_{0}$. Initial value $\dot{a}_{0}$ is supposed to be positive. Solutions $(\beta, a)$ then satisfy

$$
\begin{aligned}
& \dot{\beta}>0, \\
& \dot{a}>0, \\
& \ddot{a}>0
\end{aligned}
$$

and

$$
\frac{2}{n(n-1)} \kappa^{2} \rho+\frac{2}{n(n-1)} \Lambda-\tilde{\kappa} a^{-2}>0 .
$$

In order that $(\beta, a)$ also satisfies the first Friedmann equation, $\dot{a}_{0}$ has to be appropriately chosen, namely, such that the first Friedmann equation is valid for $t=t_{0}$, which is possible, in view of (116).

Proof. By introducing a new variable

$$
\varphi=\dot{a}
$$

we may consider a flow equation for $(\beta, a, \varphi)$, where $\dot{\varphi}$ replaces $\ddot{a}$ and

$$
\dot{a}=\varphi
$$

is an additional equation.

Then, choosing $\beta_{0}, a_{0}$ as above and $\varphi_{0}>0$ arbitrary, the flow has a solution on a maximal time interval

$$
I=\left[t_{0}, t_{1}\right), \quad t_{1}>t_{0},
$$

because of Lemma 2 and Lemma 3. It is also obvious that Relations (113)-(116) are valid in view of these lemmata.

Furthermore, if interval $I$ was bounded, then the flow would have a singularity at $t=t_{1}$, which is not possible in view of Relation (99), which would imply that $\beta, \dot{\beta}$ as well as $a$ and $\dot{a}$ would tend to infinity by approaching $t_{1}$, which, however, contradicts the second Friedmann Equation (109), from which we then would infer

$$
0<\ddot{a} \leq c a \quad \forall t \in I,
$$

an apparent contradiction. Hence, we deduce

$$
I=\left[t_{0}, \infty\right) .
$$

It remains to prove that the first Friedmann equation is satisfied if $\dot{a}_{0}$ is chosen appropriately. Define

$$
\Phi=\dot{a}^{2}-\left\{\frac{2}{n(n-1)} \kappa^{2} \rho+\frac{2}{n(n-1)} \Lambda\right\} a^{2}+\tilde{\kappa} ;
$$

then, we obtain

$$
\dot{\Phi}=0,
$$

in view of the continuity equations and Equation (109), yielding

$$
\Phi(t)=\Phi\left(t_{0}\right)=0 \quad \forall t \in I .
$$




\section{Missing Antimatter}

In [10], Theorem 4.3.1, p. 110, we proved that a temporal eigenfunction $w=w(t)$ defined in $\mathbb{R}_{+}$ can be naturally extended past Big Bang singularity $\{t=0\}$ by defining

$$
w(-t)=-w(t), \quad \forall t>0 .
$$

The extended function is then of class $C^{2, \alpha}$,

$$
w \in C^{2, \alpha}(\mathbb{R}),
$$

for some $0<\alpha<1$ and its restriction to $\{t<0\}$ is also a solution of the variational eigenvalue problem. Hence, we have two quantum spacetimes

$$
Q_{-}=\mathbb{R}_{-} \times \mathcal{S}_{0}
$$

and

$$
Q_{+}=\mathbb{R}_{+} \times \mathcal{S}_{0}
$$

and a $C^{2, \alpha}$ transition between them. If we assume that common time function $t$ is future directed in both quantum spacetimes, then the singularity in $\{t=0\}$ would be a Big Crunch for $Q_{-}$and a Big Bang for $Q_{+}$; similarly for corresponding Friedmann universes $N_{\mp}$ governed by the Einstein equations. No further singularities are present, i.e., spacetime $N_{-}$has no beginning but ends in in a Big Crunch and is recreated with a Big Bang as spacetime $N_{+}$.

This scenario would be acceptable if it described a cyclical universe. However, there are no further cycles, there would only be one transition from a Big Crunch to a Big Bang. Therefore, the mathematical alternative, namely, that at the Big Bang two universes with opposite light cones are created, is more convincing, especially if the CPT theorem is taken into account, which requires that the matter content in the universe with opposite time direction would be antimatter. This second scenario would explain what happened to the missing antimatter.

\section{Spherically Symmetric Eigenfunctions in Hyperbolic Space}

Spatial Hamiltonian $H_{1}$ is a linear elliptic operator

$$
H_{1} v=-(n-1) \Delta v-\frac{n}{2} R v,
$$

where the Laplacian is the Laplacian in $\mathcal{S}_{0}$ and $R$ the corresponding scalar curvature. We then look for eigenfunctions or, more precisely, eigendistributions $v$,

$$
H_{1} v=\lambda v,
$$

such that for each temporal eigenfunction $\left(\lambda_{i}, w_{i}\right)$ there exists a matching spatial pair $\left(\lambda_{i}, v_{i}\right)$. Product

$$
u_{i}=w_{i} v_{i}
$$

would then be a solution of wave Equation (4) on page 2.

If $\mathcal{S}_{0}$ is the hyperbolic space $\mathbb{H}^{n}, n \geq 3$, we have

$$
R=-n(n-1)
$$

and, given any temporal eigenvalue $\lambda_{i}$, we have to find functions $v_{i}$ satisfying

$$
-(n-1) \Delta v_{i}=\left(\lambda_{i}-\frac{n^{2}}{2}(n-1)\right) v_{i} .
$$


We also look for spherically symmetric eigenfunctions $v_{i}$. In hyperbolic space, the radial eigenfunctions, known as spherical functions, are well-known: For each $\mu \in \mathbb{C}$, there exists exactly one radial eigenfunction $\varphi_{\mu}$ of the Laplacian, satisfying

$$
-\Delta \varphi_{\mu}=\left(\mu^{2}+\rho^{2}\right) \varphi_{\mu}
$$

and

$$
\varphi_{\mu}(0)=1,
$$

where

$$
\rho=\frac{n-1}{2}
$$

see, e.g., [16], Section 2 and the references therein. Here, we introduced geodesic polar coordinates $(r, \xi)$ in $\mathbb{H}^{n}$, and $\varphi_{\mu}$ only depend on $r$. The $\varphi_{\mu}$ have the integral representation

$$
\varphi_{\mu}(r)=c_{n}(\sinh r)^{2-n} \int_{-r}^{r}(\cosh r-\cosh t)^{\frac{n-3}{2}} e^{-i \mu t} d t,
$$

cf. ([17], Equation (6), p. 4).

Since the $\varphi_{\mu}$ are distributions, they are smooth in $\mathbb{H}^{n}$, cf. [18], Theorem 3.2, p. 125. Furthermore, for each $i \in \mathbb{N}$, we can choose $\mu_{i} \in \mathbb{C}$, such that

$$
(n-1)\left(\mu_{i}^{2}+\rho^{2}\right)=\lambda_{i}-\frac{n^{2}}{2}(n-1) .
$$

Obviously, there are two solutions, $\mu_{i}$ and $-\mu_{i}$, but the corresponding eigenfunctions are identical as can be easily checked.

Funding: This research received no external funding.

Conflicts of Interest: The author declares no conflict of interest.

\section{References}

1. Arnowitt, R.; Deser, S.; Misner, C.W. The dynamics of general relativity. In Gravitation: An Introduction to Current Research; Witten, L., Ed.; John Wiley: New York, NY, USA, 1962; pp. 227-265.

2. Dirac, P.A.M. Lectures on Quantum Mechanics; Second printing of the 1964 original; Belfer Graduate School of Science Monographs Series; Belfer Graduate School of Science: New York, NY, USA, 1967; Volume 2.

3. DeWitt, B.S. Quantum Theory of Gravity. I. The Canonical Theory. Phys. Rev. 1967, 160, 1113-1148. [CrossRef]

4. Kiefer, C. Quantum Gravity, 2nd ed.; International Series of Monographs on Physics; Oxford University Press: Oxford, UK, 2007.

5. Thiemann, T. Modern Canonical Quantum General Relativity; Cambridge Monographs on Mathematical Physics, With a foreword by Chris Isham; Cambridge University Press: Cambridge, UK, 2007.

6. Gerhardt, C. Quantum cosmological Friedman models with an initial singularity. Class. Quantum Grav. 2009, 26, 015001. [CrossRef]

7. Gerhardt, C. The quantization of gravity in globally hyperbolic spacetimes. Adv. Theor. Math. Phys. 2013, 17, 1357-1391. [CrossRef]

8. Gerhardt, C. A unified quantum theory I: gravity interacting with a Yang-Mills field. Adv. Theor. Math. Phys. 2014, 18, 1043-1062. [CrossRef]

9. Gerhardt, C. The quantization of gravity. Adv. Theor. Math. Phys. 2018, 22, 709-757. [CrossRef]

10. Gerhardt, C. The Quantization of Gravity, 1st ed.; Fundamental Theories of Physics; Springer: Cham, Switzerland, 2018; Volume 194. [CrossRef]

11. Gerhardt, C. The quantization of a black hole. arXiv 2016, arXiv:1608.08209.

12. Gerhardt, C. The quantum development of an asymptotically Euclidean Cauchy hypersurface. arXiv 2016, arXiv:1612.03469. 
13. Gerhardt, C. The quantization of a Kerr-AdS black hole. Adv. Math. Phys. 2018, 2018. [CrossRef]

14. Capozziello, S.; Lobo, F.S.; Mimoso, J.P. Generalized energy conditions in Extended Theories of Gravity. Phys. Rev. D 2015, 91, 124019,. [CrossRef]

15. Choudhury, S.; Sen, M.; Sadhukhan, S. Can dark matter be an artifact of extended theories of gravity? Eur. Phys. J. C 2016, 76, 494. [CrossRef]

16. Anker, J.P.; Pierfelice, V. Wave and Klein-Gordon equations on hyperbolic spaces. Anal. PDE 2014, 7, 953-995.

17. Anker, J.P.; Pierfelice, V.; Vallarino, M. The wave equation on hyperbolic spaces. arXiv 2010, arXiv:1010.2372.

18. Lions, J.-L.; Magenes, E. Non-Homogeneous Boundary Value Problems and Applications; Kenneth, P., Trans.; Die Grundlehren der mathematischen Wissenschaften, Band 181; Springer: New York, NY, USA, 1972; Volume I.

(C) 2019 by the author. Licensee MDPI, Basel, Switzerland. This article is an open access article distributed under the terms and conditions of the Creative Commons Attribution (CC BY) license (http://creativecommons.org/licenses/by/4.0/). 